\title{
FOOD PATTERNS AND EATING HABITS AMONG CHILDREN DURING THE PANDEMIC: A PARENT'S PERSPECTIVE
}

\author{
Dr. Vaishali Patil \\ Senior Associate Dean- Research and Publication \& IT, Prin. L.N. Welingkar Institute of \\ Management Development \& Research, Mumbai \\ Dr. Chaitra Khole \\ Assistant Professor, Prin. L.N. Welingkar Institute of Management Development \& Research, \\ Mumbai
}

\begin{abstract}
The current pandemic of the novel COVID-19 lead to the nationwide lockdown for a relatively long period. The lockdown has led to changes among every individual at different level. Although children are less severely infected with mild infectious, they are still at risk. One of the major strategies for prevention of the spread of the disease was the 'nationwide lockdown' which was adopted by Government. One of the outcomes of the lockdown was disrupted schedules of many individuals. It also leads to irregularity and hindrance for 'food access' toindividuals which further affect the overall household. One such subgroup which have been affected are the children. With changes in their lifestyle and timetable there is indeed a change in their eating habits and food consumption. Indepth interviews were conducted with 9 parents in Pune and Mumbai city. They described their experiences of children ranging 2 years up to 18 years. There were five major themes which emerged from the study: effect of pandemic on daily lifestyle, dietary changes at household level, eating patterns and food practices among children and lastly activities of children. In this paper viewpoints and experiences of parents on their children's food eating habits and practices during COVID-19 pandemic are presented.
\end{abstract}

KEYWORDS: COVID-19, Children, Food patterns and habits

Article Received: 10 August 2020, Revised: 25 October 2020, Accepted: 18 November 2020

\subsection{BACKGROUND OF THE STUDY}

India is experiencing a phenomenon which includes the current epidemiologic transition leading to NCDs, the environmental transition which leads to communicable diseases and the current burden of diseases and this collective phenomenon is known as triple burden of diseases which includes communicable, noncommunicable, emerging and re-emerging infectious diseases. There is also a twin epidemic of continuing/ emerging infectious diseases along with degenerative diseases. This condition of the country is a 'dilapidated' state with major public health problems like under nutrition, malaria, tuberculosis, high maternal, child mortality, safe drinking water and sanitation and one of the most important pandemic issues. Various lifestyle diseases which are caused due to change in living habits and lifestyle modifications are diabetes, cardiovascular diseases, cancer, typhoid, and other water-borne diseases (Public Health in
India: Issues and Challenges, 2016). The rise of Non-communicable diseases (NCDs) than infectious diseases elicits the causal effect of lifestyle and nutrition status.

The year 2020 has been one of the year which will always be remembered by mankind. The most important attribute of this year was the spread of the novel corona virus in the country post its emergence in 2019. The first case was reported on $30^{\text {th }}$ January 2019 . This disease has affected the entire world in various capacities and trajectories. India is the fifth largest country in terms of the highest number of confirmed cases, as of $31^{\text {st }}$ January 2021 India has reported 10,746,183 confirmed cases and a rise of 13,052 cases on $31^{\text {st }}$ January 2021 (Novel Coronavirus Disease (COVID-19) Situation Update Report-53, p. 2021). The numbers of COVID-19 cases in India are not uniformly distributed; rather there are some hotspots where there are maximum numbers of COVID-19 positive cases. The major hotspots 
states were Maharashtra, Tamil Nadu, Delhi, Gujarat, and Rajasthan, respectively. Data suggests more than $33 \%$ of the total COVID19 cases were documented in Maharashtra, followed by $11 \%$ in Tamil Nadu, $9 \%$ in Delhi, $8 \%$ in Gujarat, and $4 \%$ in Rajasthan. More than $60 \%$ of the total COVID-19 cases were recorded from these five states in India. In the State of Maharashtra since the beginning the numbers have been on a higher side. From 14,541 cases in Maharshtra in the month of May 2020. Maharashtra has reported the highest number COVID19 cases.

In response of the global COVID 19 pandemic, the Prime Minister announced Janata (curfew) on $22^{\text {nd }}$ March 2020 followed by the complete nationwide lockdown for a period of 21 days (Singh \& Chauhan, 2020). One of the key preventive measure followed worldwide and also in India has been social distancing and 'lockdown' (Marbaniang, 2020). A lockdown can be described as an emergency protocol that prevents people from leaving a given area, allowing essential supplies, grocery stores, pharmacies and banks to serve people and non-essential services to remain shut for the entire period (What a coronavirus lockdown looks likes, and whatyou can do \& what you can't Read more at:https://economictimes.indiatimes.com/news/ politics-and-nation/coronavirus-outbreakwhat-a-lockdown-will-look-like-

foryou/articleshow/74760719.cms?utm_source =con, 2020)

This sudden lockdown affected various age groups. The elderly being at highest risk in the beginning the further widespread of this disease, lead to increase in vulnerability like in various age groups like the adults and also a considerable proportion among the younger population. Some of the key issues of the pandemic are unemployment, unprecedented increase in poverty, poor physical and mental health. It has also affected food, nutrition and health security of vulnerable populations including children, pregnant and lactating women underpinning various social and health inequalities.

\subsection{REVIEW OF LITERATURE:}

The COVID 19 pandemic has led to lifechanging challenges among people across the globe. Terms like "social distancing" and "self- isolation" have become a reality. As people are trying to get accustomed with this, the confinement has drastically impacted citizens' lives bringing about a sudden and radical change in their daily routine and lifestyle.Social isolation for extended periods can lead to boredom and stress which may further subject individuals to develop a greater tendency of overeating especially "comfort foods" which are usually high in calories. Moreover, prolonged confinement at home may induce an increased screen time and reduced outdoor time thus, augmenting sedentarism. Apart from this, being homebound and facing disruptions in daily routines may affect the sleeping patterns as well as the quality of sleep (Kumari, et al., 2020). In this study food and nutrition security has been attempted to be accessed by understanding "access to food" which is explored by understanding various food patterns and practices followed by children, using a parents perspectives. An extensive review of literature helps in understanding interplay of various factors interplaying affecting factors. In a study the NFHS-4 (2015-2016) data was assessed to study the impact of anaemia and nutritional status on COVID-19 cases over the hotspot states of India. The study was performed over more than $60 \%$ of COVID-19 cases were recorded. The results of the study showed that adults with below normal BMI, overweight or obesity, and anaemia are the most vulnerable to COVID-19 and other respiratory disorders (Das, Das, \& Ghosh, 2020). In another study the author summed up that one of the main outcome of the pandemic was deterioration of dietary habits impacting health systems worsening NCDs (Jayawardena \& Misra, 2020). In another study the author suggested weight gain and risk of type 2 diabetes mellitus (T2DM) during lockdown in the current pandemic (Ghosal, et al., 2020)

There was a study conducted in North India to assess the effect of lockdown on lifestyle and other medical issues of patients with type 2 diabetes. Results showed that snacking consumption has increased by 23 percent, 42 percent reduction in exercise and 19 percent had weight gain (Ghosh, Arora, Gupta, Anoop, \& Misra, 2020). A study was carried out to access weight changes and food patterns during the pandemic among the type 2 diabetes patients in which 56 percent reported 
to have no changes in dietary intake whereas 25 percent reported to eating less than their intake. The patients also reported about irregular meal timings and increase in carbohydrate and fat in their diet. There is no doubt that COVID- 19 has become a natural experiment illustrating how unprepared the world is to protect populations against hunger, food, nutrition, and health insecurity during global emergency situations. As a result of rapid unplanned urbanization, and climate change, these types of pandemics will likely be with us for the years to come (Jowell, Carstensen, \& Barry, 2020)

\section{EFFECT ON THE CHILDREN}

In another study the author stated the pandemic is likely to affect the food security as there is decline in access to sufficient, safe and nutritious food to meet dietary needs and food preferences for an active and healthy life. The author also discussed household food insecurity (HFI) to affect behavioural problems, poor academic performance and intellectual outcomes, risk of chronic undernutrition, obesity and infectious diseases among children. Thus a'syndemic' paradigm with two or more coexistent diseases cause excess burden of disease in a population (Pérez- Escamilla, Cunningham, \& Moran, 2020).

The coronavirus disease (COVID-19) pandemic has multifaceted impacts on children, that is psychological, mental, physical, social and cultural (Dalton, Rapa, \& Stein, 2020). The younger population (>18 years) are also affected by the pandemic (The New Indian Express, 2020). Looking retrospectively the pandemic had a major psychological, physical, social and cultural impact on the children, considering about forty one percent population to be lesser than 18 years understanding impact on their health is crucial. During the lockdown many children lacked physical activity and socialising which made them lead a monotonous life with irritation and frustration. They also face issues like anxiety, mood swings, insomnia and loss of appetite (Kumar, Nayar, \& Bhat, 2020). Contrary to the general perception that the novel coronavirus spares children, there have been reports of children below 12 years of age becoming infected in India, although compared to other age groups far fewer are affected (The New Indian Express, 2020). Agencies working on children's issues have also expressed their concern that heightened anxiety and stress on families due to COVID19 may lead to an exacerbation of mental health problems in children (APA, 2020).

A large study was conducted in India to assess impact of COVID-19 on lifestyle related behaviours and it was found that there was improvement in healthy meal consumption patterns and restriction of unhealthy food, reduced physical activity and increase in daily screen time was observed in the younger population. There was also induced stress and anxiety among the sample (995 respondents) (Chopra, et al., 2020)

A review was conducted to assess the dietary patterns in India and its association with NCDs. Data was assessed using various databases with population subgroups ranging from children to adults. The various food groups in different states varied from 7 to 104 . About 29 dietary patterns which were observed and some of the common food patterns included vegetarianism, the results also emphasized on the choice of food, which included fruits, vegetables, pulses and cereals, and dairy products. Interestingly food groups including fruits, vegetables, rice, and pulses were predominantly seen in the North and Western region. Sweets and snacks were more characteristic to East and South with high consumption of meat and fish. The association dietary patterns and practices on NCDs were reflected on hypertension, diabetes, and cholesterol (Green, Milner, Joy, \& Sutapa, 2016). A study in south India reveals parents awareness and concern especially for their children's health during COVID times (Palanivel, Gayathri, Gheena, \& Vishnupriya, 2020). The reason behind the lower prevalence of COVID-19 among children compared to adults is still unclear. Latest studies suggest that less exposure, less vigorous immune response, and incomplete functionality of Angiotensin- Converting Enzyme II (ACE2) in children - as the potential receptor for SARSCoV2 - are responsible for the significantly fewer severe cases in children. Despite this there is still possibility of transmission (Dong, Mo, Hu, \& Jhiang, 2020) 
India being a rich and diverse country has various cuisines which are associated with the social identity of individuals, religion, region, cultural factors, agricultural factors, consumer patterns, and dietary transitions. A number of factors like cultural, psychological, lifestyle and food trends affect the food decisions of any individuals. The cultural factors include food habits which are developed among individuals are in regard to a culture or tradition, which in turn determine ones attitude towards specific food. Psychological factors include food preferences, likes and dislikes and sensory attributes of food. Lifestyle factors affect food selection and lastly, food trends depend on taste, convenience, a fusion of various flavours and fads.Due to lockdown, many children have had no physical access to friends, peers, schoolmates and relatives for over two months. Limited or no opportunity for outdoor play and socialization may also impact children adversely, making them easily bored, angry and frustrated. Although many are active on mobile phones and virtually connected, there is higher probability that they will become increasingly engrossed in social media and online entertainment. Maharashtra being the third largest state of the country with highest number of COVID-19 cases it was observed that Pune and Mumbai had the highest number of cases (Kodge, 2020). It is thus important to explore the effect of this pandemic on the food and dietary patterns of the children in these areas as above literature suggests a complicated picture of the status. There are limited understanding in this field by researchers across the world. Hence the main objective of this study was to understand dietary changes especially among the children through the parent's perspective and its effect on their overall day to day life.

\subsection{RATIONALE AND OBJECTIVES OF THE STUDY}

With this background a study was undertaken to examine the dietary intake habits and food patterns of children through the parent's perspective considering the nationwide lockdown in the COVID times. The objective of the study was to explore perspectives of parents regarding food patterns among children during the pandemic

The specific research questions of the study are as follows a. What are the food preferences of the children?

b. What is the various food eating habits that have been formed over the last few months?

c. What are the dietary changes observed among the children?

\subsection{METHODOLOGY}

The study was conducted among parents living in Pune and Mumbai. This area has been purposely selected because COVID 19 cases were very high and the study mainly aims at understanding dietary intakeand food pattern among the children in this pandemic. This study was shaped underpinning secondary sources like various studies and literature review in the last one year. Both primary and secondary data sources are further triangulated. A qualitative interpretivist approach was used in the study as the nature of this mode of inquiry seeks to understand a phenomenon within cultural and social context in the natural setting (Cresswell, J. 2008). Considering the critical situation of Maharashtra a survey based study was not feasible thus a 'qualitative research paradigm' has been used in the study. A purposive sampling method was used and through snowball sampling online data was collected through telephonic interviews from 9 parents residing in Pune and Mumbai. In depth interview method was used for carrying out the study. This study is based on qualitative data to explore parents' perspectives.

\subsection{TOOLS OF DATA COLLECTION}

Data collection was done by audio recorded individual interviews primarily telephonic in nature expect four which were done face to face. Semi-structured interview schedule with open ended questions were mainly used to collect information for the study. The questions covered participants perception and experiences of food patterns and eating behaviors of their respective children during the pandemic. The interview schedule was mainly based on understanding effect of pandemic in daily lifestyle among people followed by dietary changes and food habits and patterns among children. The interview schedule was based on review of literature which captured similar objectives.

\section{Participants}


Data was collected from 9 participants, which were recruited in the study using purposive and snowball sampling. Maximum variation strategy was used in recruitment of the participants to ensure diversity in views and experiences. The participants ranged from income groups (IG) (low, middle and high) and education status (uneducated and educated)

\subsection{ANALYSIS}

In this specific study data analysis and data collection were simultaneous processes where did was gathered, shaped and analysed while recruiting newer participants. The qualitative enquiry would be in the form of narratives which would be further transcribed and translated to English. Coding was done to check for emerging themes from the data. A preliminary analysis of the in depth interviews was done by identifying major themes emerging from the data. Depending on the transcriptions themes would be generated based on the research objectives and research questions and further categories and recategorized to form larger themes and subthemes.

\subsection{RESULTS AND FINDINGS}

The results and findings are based on the research questions and 4 themes have emerged from the data. The themes identified are discussed below and their content is illustrated with quotes from the participants. The section starts with understanding the daily lifestyle of the participants in general; followed by understanding changes in their children.

\subsubsection{Effect of pandemic on daily lifestyle}

The pandemic affected everyone in different capacities. Among many people their social circle was affected as there was limited socializing and meeting people. It also affected mentally, as with the disease progression and increase in the number of cases each day there was a constant fear and stigma among people. It also affected 'individual' physical space as everyone was bound to be together in a confined space. In this study when it was questioned the effect of pandemic in their daily lifestyle almost all the participants answered that the pandemic drastically affected their life. Schools and colleges till today (dated 2/2/21) have not been completely functionalwhich has affected the education calendar of all the students.In this study some of the notable observations were, that many parents refrained from sending their children to school, many of the parents work from home and some had also moved to their native places during the early phase of the lockdown. All the parents faced a severe change in their daily life during the lockdown phase. One of the participants mentioned the following

"Due to school there was a set routine for everyone now it's kind of disturbed" (LIG, 36)

The above response was from a participant who was from a low-income group; she had three children living in tenement with six other family members. All the children went to school in the morning followed by the 'parents' who went out to work (daily wage earning husband and informal labour). But in the current situation all the children and family members lived together in a confined space. The class timings of the children clashed with each another and having limited sources for online learning this setting was a difficult situation for the participants in terms of managing their daily timetable. There was another participantwho was aworking professional living in Mumbai who said she was feeling relieved during the lockdown the participant said,

"Work load and morning chaos has reduced" (HIG, 40)

The participant had been living in Mumbai for more than a decade with an erratic schedule. The lockdown gave a 'breather' to sit back and also give time to the family members during the pandemic. As we know there is a constant environmental distress owing to fluctuation owing to industrialization and rise in population, a phenomenon more in case of cities like Pune and Mumbai this leads to higher susceptibility to infectious diseases and other chronic air borne diseases. Work from home has been one of the good practices followed in the pandemic which was a good preventive measure. Another angle to this study was a participant who mentioned the following

\section{"Health has improved" (HIG, 40)}

With constant travelling and exposure to the environment rendered with constant health issues and stress. The lockdown wherein the mobility was reduced helped in improvement of health. The next theme which had emerged 
was changes among the family members. As we have already seen above most of the participants mentioned having a disturbed time table in their daily life. One of the participant mentioned despite having a disturbed time table but the positive part of this pandemic was spending time with the family members. Some of the responses of the participants are as follows

"Time table for the entire family has disturbed but it has also given time to spend with the near and dear ones" $(M I G, 30)$

In this study it is observed that the pandemic and lockdown was normalized after few months. Another participant mentioned on changes in the lifestyle, followed by adapting and adopting to a new lifestyle in this pandemic. It is observed that a sense of adjustment and acceptance of change was normalized by all the individuals. She said the following

"Initially it took some time to adjust with everyone being around and managing everything at home but now everything is sorted in a new routine" $(H I G, 32)$

\subsubsection{Dietary changes at the household \\ level}

With nutrition transition which the country has experienced for the last few decades there are changes in the patterns of food consumption. Food consumption among children in this study is explored by understanding dietary intake and various changes associated with the intake. With urbanization the current generation has adapted and adopted nutrition transition and consumption of food not just regular home-made meals but also from other sources like eateries which has been the new normal. During the lockdown period many individuals faced challenges as ready to eat and takeaways had been shut so everyone depended on home cooked food. Other phenomenon which is often observed in the last few months was cooking various cuisines served at eateries. This was done by following YouTube videos of various famous chefs. Many of the respondents of this study mentioned on exploring and experimenting cooking. One of the participant said the following
"Consumption of outside food has stopped. But we make a lot of recipes at home which at times are not that healthy" $(H I G, 32)$

In the context of home cooked food, experimenting the culinary skills and maintaining balance in variety of cooking a lot of junk food under the pretext of 'healthy' was made and consumed by many families. After few months of the lockdown when the rules were made flexible there was again normalcy with regard to consumption of refined and ready to eat meals. This means despite people were not going outside to eateries but consuming different form of processed and fermented food at home. One of the participant said the following

"Unfortunately, there is little increase in refined food like bakery items and ready to cook meals as they are easier to make" (HIG, 33)

With work load, work from home schedules, timetable disturbances and various other factors relying and consuming ready to eat meals and bakery items was on a rise.

\subsubsection{Factors Affecting Dietary Intake}

\section{Physical activity engagement}

In the pandemic with lockdown rules, social distancing, vulnerability among children and stigma of the disease had a major impact on their physical activity. With online learning modules their physical activity was limited even more. In this study when the participants were asked on the children's physical activity engagement the following responses were given,

\section{“Doesn't go out to play at all" (HIG, 40)}

Children were initially conditioned to stay indoors and this made them habitual of staying back reducing their physical activities. Some of the participants had shifted to their native where the impact of COVID19 lesser as compared to that of largely populated cities. One of such participant reported the following,

"Consciously include physical activity in their daily life, as we were in the native throughout the lockdown there was more space and children enjoyed and indulged in various games" $(H I G, 30)$

The above participant mentioned allowing the children to play as they lived in the natives 
where there were lesser cases and much more space as compared to that of a city. So the children lived in joint families played in the adjoining area of the house. There were also some conscious participants (parents) who tried to include some physical activity in their life in various forms. One of the participant reported the following,

"Recently my daughter does cycling in society premises" (HIG, 33)

The participant was aware of the reduced physical activity and need of physical activity for their children, hence cycling in the society prices on few days a week was followed. The next section discusses screen time which was followed by the children.

\section{Screen time}

Studies suggest impact of screen time on food consumption pattern among the children. Some of the parents mentioned the children didn't want television but one very interesting response was given by one parent. She said the following

"Screen time has definitely increased considering online mode for school and other classes" (HIG, 35)

With the current education innovation and online learning mode children used computers and phone for not just learning but also to submit their assignments and submissions which lead to more and more time spend on computers and mobile phones. Thus, all the children were exposed to high screen time throughout the pandemic

\section{Hours of sleep}

Studies suggest a relationship with regard to hours of sleep and food patterns in adults. A participant said the following

\section{"Sleep pattern has been disturbed" (HIG, 30)}

In this study many parents reported that with change in the timetable the sleeping patterns have also changed, and many a times children sleep till late mornings and are awake late nights.

\subsubsection{Food patterns and practices Skipping meals}

The next aspect discussed in this study is skipping meals. As already seen in the above sections due to disturbed timetable many parents reported on children missing and skipping their meals. One of the participants said the following statement,

"My children miss their meals everyday till today, they don't wake up on time miss their breakfast, when we leave for work, they don't eat cooked meals instead have a Rs. 10 Maggi or have Kurkure" (LIG, 37)

In such kind of situation, the severity of the problem not just skipping meal but also access and choice for having nutritious food, thus in such a scenario a question food security arises. Many families and also children faced food security issues during the lockdown. Among most of the participants the children missed their breakfast due to change in the timetable, one of the participant said the following statement,

\section{"Breakfast was skipped, rest of the meals are} on time" $(I G, 30)$

Whereas one of the participant mentioned taking an initiative to maintain the meals and meal timing of the children

"Not really, I skip meals but make sure kids maintain their meals along with the timetable" (HIG, 32)

\section{Snacking pattern and consumption of junk food}

This section discussed on the snacking patterns seen among the children. Most of participants mentioned that children generally had ready to eat snacks, easy to cook meals or fruits as snacks items. One interesting similarity food among children was their affinity to 'Maggi', most of the participants mentioned Maggi as a part of their diet. During the pandemic in the lockdown phase could not have access to markets and thus many parents made snacks at home, one of the most popular was the bakery items. Some of the responses on their snacking patterns are as follows,

"Maggi for snacks" (HIG, 30)

"Children love homemade pasta and pizza so I watch YouTube and make various recipes" (MIG, 35)

One of the participant tried to inculcate healthy snack items like dry fruits in the diet.

I give healthy snacks like nuts and dry fruits (HIG, 32) 
Further in this section the parents were questioned about their intake of junk food. To begin with consumption of junk food is no longer an option but a norm which is consciously and subconsciously consumed by everyone in the society. There is bigger umbrella wherein the children from all sections of the society preferred junk food as a part of daily diet. One of the participant belonging to the LIG stated the following,

"My kids are eating junk (out of the packet food) on everyday basis, Maggi is what they are eating a meal, if not that then Kurkure or chips" (LIG, 37)

Another participant from a HIG stated the following,

"Junk food in the diet has definitely increased. My daughter has bread and mayonnaise for breakfast its her favorite. Other includes Maggi, French fries, chips, pizza" (HIG, 35)

One of the participant had recently moved back to India and she stated the following,

"My son is just 3 years old but he doesn't eat anything, he dislikes traditional food including dal rice, thus we give him soups, Maggi or cheese cube, we were in the states before so he currently dislikes Indian food" $(\mathrm{HIG}, 33)$

\section{Intake of sweetened beverages}

After exploring snacking and junk food consumption patterns intake of beverages was assessed. Besides homemade beverages many children like to have sodas and other sweetened beverages which contribute to 'empty calories' in their diet. In this study most of children enjoyed having outside beverages and consumed more than twice a week. Some of the responses of the participants were as a follows

"Sometimes my son has a tetra pack of juice" (HIG, 40)

"Mirinda is her favorite" (HIG, 37)

Regardless one of the participant of the study was conscious with the choice of beverages and its ill effects on the child mentioned that since the beginning she included fruits in the diet and beverages were never given due to which now the child doesn't prefer consumption of any sweetened beverage.

"No interest in having beverages" (HIG, 30)

\section{Intake of sweets}

This section discusses intake of sweets. It is a preconceived notion that children like sweets. All the participants reported that their children consumed sweets on regular basis. Some of the following are the responses.

"To be honest there is a very high chocolate consumption among the kids, even my nephews and nieces they have a lot of chocolates" (HIG, 30)

It is observed from the above response that intake of chocolates is a normal phenomenon among kids. Parents and relatives of the children would give chocolates to the children on regular basis. The respondent also mentioned, they got chocolates whenever one went out of the house.

"Initially I was giving one chocolate per day now I have changed that to 2-3 per week" (HIG, 32)

The above participant used to give chocolates on daily basis as the children would demand for it and while managing children, however in the lockdown the mother had reduced the number of chocolates per week.

Another participant mentioned the following,

"I used to give chocolates, but my son get stomach infections and he becomes hyperactive that's why I don't give him too much sweets" (HIG, 33)

The participant mentioned their child to get 'worms' as a result of having sweets, thus only fruits was given instead of chocolates. One interesting finding in this study was wherein the participant mentioned having desserts.

If not chocolates kids demand sweet items like brownie, cake to be made at home (MIG, 35)

As already mentioned in the above section many parents got used to cooking and experimenting recipes from online medium, various foodstuffs from eateries was made available to the children through home cooking.

\section{Meals portions}

With reference to the above sections, it was observed that regular time table leading to skipping meals lead to hunger further leading a larger meal as it had to compensate the previous skipped meal. Many participants mentioned that the children had bigger portion 
of meal than what they had earlier before the pandemic. Some of the responses are as follows,

"Food intake has improved, sometimes there is overeating" (HIG, 30)

"After skipping meals portion size for the meal is more" (MIG, 36)

Whereas a participant mentioned there has been no change in the portion size of the meal.

"Not much" (HIG, 32)

\section{Balanced diet}

The parents were then asked about their perception on balanced diet and consumption of balanced diet by the children. As this study was done in the urban areas of Maharashtra and many of the participants were educated they fairly had a good idea about balanced diet and its importance in one's diet. Some of the following responses were given by them,

"In the pandemic there is an increase in intake of eggs and mutton which was not before" (HIG, 30)

In the above case one of the participants mentioned of not getting good quality non vegetarian food items in cities as compared to that of villages. Thus, in the lockdown when their family lived in their native had access to better quality non-vegetarian items than the ones which are mass produced for the cities by poultries.

One of the participants who is self-taught about nutrition mentioned

\section{"I give different variety of food" (HIG, 32)}

She had the knowledge of including various food groups in the diet. Along the similar lines one more participant mentioned on being aware about inclusion of food groups in the diet.

"I include food items from each food group in their diet, I have seen videos of healthy eating on YouTube" (MIG, 37)

\section{Tradition food items}

While studying various food pattern trends it's important to also discuss traditional patterns which are based on social dimensions. As this study was carried out in the urban areas and assuming there would be lot of transitions in one's diet it's also important explore what are the traditional items which are still eaten by the household and the children. One of the participantsaid the following,

"Bhakril has been introduced to our diet on daily basis, it is easier to digest" (HIG, 40, $H I G, 35)$

Many participants mentioned having Bhakri in their diet as its easier to digest as during the lockdown, many people were working from home with minimum physical activity. One of the participant also mentioned on having traditional food items like a mix of vegetables and meat and various soups to improve immunity.

"Traditional non vegetarian items are now introduced to our diet" (HIG, 30)

One of the participant mentioned some of the snack items which were made and included in the diet for the children instead of junk food.

"Ghavan'andbajriwade are made for the kids" (MIG, 35)

\section{Immunity boosting foodstuffs and supplements}

The whole pandemic shook the nation and people tired various home remedies for better health. People followed various sources for information to improve immunity and reduce chances to get infected. Children being one of the post vulnerable population it was observed that it is challenging for the parents. One of the parents mentioned the following,

"I am giving calcium supplements and increased milk in their diet. I have observed the immunity has increased, there are lower hospital visits and definitely lower infections" (HIG, 40)

One of the famous fads followed during this time is immunity boosters in the form of Kadhas

"Masala Kadha" " (HIG, 35)

${ }^{1}$ Bhakri is a round flat unleavened bread often used in the cuisine of the state of Maharashtra

${ }^{2}$ Ghavan is a traditional breakfast recipe from Konkan region made up of brown rice flour

${ }^{3}$ Bajriwade is a recipe made from bajri millet along with wheat flour and made into small flat round pancakes which are deep fried 
"We make kadha of dalchini", lavang ${ }^{6}$ and chai pati ${ }^{7}$ we also have haldi ${ }^{8}$ pan for boosting our immunity" (HIG, 30)

Some parents also gave supplements like chewable vitamin $\mathrm{C}$ to the children for improving the immunity.

\section{Weight changes among children}

There were diverse responses when questioned about weight changes among the children, the following are some of the responses.

"There is weight loss among all my three children" $(L I G, 37)$

"Increase in weight" (HIG, 40)

"Due to acidity there is weight loss" (HIG, 42)

"Health has improved and there is increase in the weight" (HIG, 30)

"Weight has reduced" (HIG, 35)

From the above responses it can be observed that due to reduced physical activity and imposition to be at home made the children inactive and also lead to a considerable increase in weight. Some of the parents who were conscious tried to incorporate physical activity in the children leading to maintained weight. Whereas with irregular timetable and lower intake of food had led to weight loss among some children.

\subsubsection{Eating Patterns among the Children}

In this section the participants discussed various food patterns among children. Some parents discussed their normal meals and changes with regard to the COVID times. A participant mentioned the following,

"I am trying to give them simple homemade food but at times biscuits and bakery items are given" (HIG, 32)

${ }^{4} \mathrm{Kadha}$ is traditional, homemade, aromatic drink which is healthy and has numerous healing properties

${ }^{5}$ Dalchini is a Marathi word for cinnamon

${ }^{6}$ Lavang is a spice (clove in English)

${ }^{7}$ Chai patiare tea leaves

${ }^{8} \mathrm{Haldi}$ is spice yellow in color otherwise known as turmeric
As children were habituated of eating from certain food groups intake of those food items were given owing to ease.

One of the participant who had just moved back to India before the lockdown gave a very different insight. She mentioned the following,

"My son has forgotten all the traditional home made food he used to have at home as he goes to day care centre" (HIG, 33)

The son used to eat everything before but as the parents were busy with their jobs the son was admitted to a day care centre which gave regular meals on specific timings. The child consumed three to four meals due to which he was subjected to a certain diet. Later during lockdown the child was not ready to eat any home cooked food.

\subsection{CONCLUSION}

The pandemic which was characterised by quarantine restrictions, social distancing, schools and work places closing, ban on group gatherings has the affected lifestyle of the population.

COVID-19 preventive measures like home quarantines, self-isolation and community lock down leads to risk factors leading to less physical activity, improper diets (Ambati \& Sucharitha, 2020). COVID-19 has also led to stressful situation of constantly updating with the details about the pandemic staying at home, further pushing people to consume more comfort foods which necessarily are not healthy which leads to food cravings and leading to multiple health issues. A recent study discusses 'emotional eating' which refers to a drive to eat a reaction of stress which is an outcome of the anxiety, stress and depression during the pandemic (Renzo, et al., 2020). In the present study there was very little physical activity by the children which is one of the reasons for weight gain among them, another observation of this study was that despite there is improvement in terms of eating homemade food along with all the family members together; there is also higher intake of homemade energy dense food with higher carbohydrates, fats, and proteins among children. Another observation of this study was intake of supplements and home remedies to increase immunity to combat the disease. The poor dietary habits along with unhealthy lifestyle can cause serious health implications 
not only among the children but can thereby affect their health in the long run. The COVID pandemic is one of the major reasons for various mechanisms to cause global malnutrition as there is serious decline in access to nutritious food and this low quality of dietary intake further affecting health of the population at large. From this study it is evident that there is mixed effect of preventive measures adopted by parents leading improvement of eating homemade food and reduction of unhealthy food but also various food eating practices which needs major changes. It is thus very important that the Government regulatory bodies consider need for expand knowledge about healthy eating habits to parents and children by having various activities in schools, campaigns, workshop and nutrition education programmes to educate the masses on basic idea of good nutrition and practices during such pandemic times. Health organisations along with educational authorities should emphasize on increasing physical activities in the form of "physical activities at home" to prevent good health and weight gain.

\section{ACKNOWLEDGEMENT}

We would like to thank each participant of this study who gave us their time

\section{CONFLICTS OF INTEREST}

The authors declare no conflict of interest

\section{REFERENCES}

- Renzo, L. D., Gualtieri, P., Cinelli, G., Bigioni, G., Soldati, L., Attina, A., . . . Rampello, T. (2020, July 19). Psychological Aspects and Eating Habits during COVID-19 Home Confinement: Results of EHLC-COVID-19 Italian Online Survey. Nutrients, 12, 1-14.

- R, S. S. (2016, July). Public Health in India: Issues and Challenges. Internation Research Journal of Interdisciplinary \& Multidisciplinary Studies (IRJIMS), II(VI), 29-36.

- Green, R., Milner, J., Joy, E. M., \&Sutapa, A. (2016, July). Dietary patterns in India: a systematic review. British Journal of Nutrition, 116(1), 142148.

- $\quad$ Singh, R. P., \& Chauhan, A. (2020, July 7). Impact of lockdown on air quality in
India during COVID-19 pandemic. Air Quality, Atmosphere \& Health, 921-928.

- Kumari, A., Ranjan, P., Vikram, N., Kaur, D., Sahu, A., Dwivedi, S., . . . Goel, A. (2020, November- December). A short questionnaire to assess changes in lifestyle-related behaviour during COVID 19 pandemic. Diabetes \& Metabolic Syndrome: Clinical Research \& Reviews, 14(6), 1697-1701.

- Kumar , A., Nayar, K., \& Bhat, L. D. (2020, May 26). Debate: COVID-19 and children in India. Child and Adolescent Mental Health, 25(3), 165-166.

- Ghosal, S., Arora, B., Dutta, K., Ghosh, A., Sinha, B., \&Misra, A. (2020, September- October). Increase in the risk of type 2 diabetes during lockdown for the COVID19 pandemic in India: A cohort analysis. Diabetes \& Metabolic Syndrome: Clinical Research \& Reviews, 14(5), 949-952.

- $\quad$ Pérez- Escamilla, R., Cunningham, K., \& Moran, V. H. (2020, May 26). COVID- 19 and maternal and child food and nutrition insecurity: a complex syndemic. Maternal \& Child Nutrition, 16(3).

- $\quad$ Novel Coronavirus Disease (COVID-19) Situation Update Report-53. (2021, February 1). Retrieved February 2021, from World health organisation: https://cdn.who.int/media/docs/defaultsource/wrindia/situation-report/indiasituation-report53.pdf?sfvrsn $=49 \mathrm{c} 97 \mathrm{~b} 8 \mathrm{~d} \_4$

- Marbaniang, S. P. (2020). Forecasting the Prevalence of COVID-19 in Maharashtra, Delhi, Kerala, and India using an ARIMA model. Retrieved from Research Square: https://www.researchsquare.com/article/rs $-34555 / \mathrm{v} 1$

- What a coronavirus lockdown looks likes, and what you can do \& what you can't Read more at: https://economictimes.indiatimes.com/ne ws/politics-and-nation/coronavirusoutbreak-what-a-lockdown-will-looklike-foryou/articleshow/74760719.cms?utm_sour 
ce=con. (2020, March 25). Economic times, India.

- Kodge, B. G. (2020, July). A review on current status of COVID19 cases in Maharashtra state of India using GIS: a case study. Nature Public Health Emergency Collection(3), 1-7.

- Das, A., Das, M., \& Ghosh, S. (2020, June 9). Impact of nutritional status and anemia on COVID-19: Is it a public health concern? Evidence from National Family Health Survey-4 (2015-2016), India. Public Health(185), 93-94.

- Ghosh, A., Arora, B., Gupta, R., Anoop, S., \&Misra, A. (2020, SeptemberOctober). Effects of nationwide lockdown during COVID-19 epidemic on lifestyle and other medical issues of patients with type 2 diabetes in north India. Diabetes \& Metabolic Syndrome: Clinical Research \& Reviews, 14(5), 917-920.

- Jayawardena, R., \&Misra, A. (2020, June 30 ). Balanced diet is a major casualty in COVID-19. Diabetes \& Metabolic Syndrome: Clinical Research \&Reviews(14), 1085-1086.

- Chopra, S., Ranjan, P., Singh, V., Kumar, S., Arora, M., Hasan, M. S., . . B Baitha, U. (2020, November- December). Impact of COVID-19 on lifestyle-related behaviours- a cross-sectional audit of responses from nine hundred and ninetyfive participants from India. Diabetes \& Metabolic Syndrome: Clinical Research \& Reviews, 14(6), 2021-2030.

- Palanivel, N., Gayathri, R., Gheena, S., \&Vishnupriya, V. (2020, October 20). Knowledge and Awareness in Maintaining Good Health During Infection Outbreak (COVID-19) Among Parents of South Indian Population - A Survey. International Journal of Current Research and Review, 12(19), 248.

- Dong, Y., Mo, X., Hu, Y., \&Jhiang, F. (2020). Epidemiological characteristics of 2143 pediatric patients with 2019 coronavirus disease in China. Retrieved from

https://pediatrics.aappublications.org/ content/pediatrics/early/2020/03/16/peds. 2020-0702. full.pdf.
- Ambati, K., \&Sucharitha, K. (2020). AN OVERVIEW OF BALANCED DIET ON COVID-19. Journal of Information and Computational Science, 13(10), 7-13.

- Jowell, A., Carstensen, L., \& Barry, M. (2020). A life-course model for healthier ageing: lessons learned during the COVID-19 pandemic. Retrieved March 2021, from Lancet: https://www.thelancet.com/journals/lanhl/ article/PIIS2666-7568(20)300088/fulltext(n.d.). 